\title{
Protective Effects of 20(S)-Protopanaxtriol on Viral Myocarditis Infected by Coxsackievirus B3
}

\author{
Xiaoyan Wang ${ }^{a, b}$ Yafeng Wang ${ }^{a, b} \quad Z^{2}$ Ren ${ }^{a, b} \quad$ Chuiwen Qian ${ }^{a, b} \quad$ Yicheng Li $^{a, b}$ \\ Qingduan Wang ${ }^{c}$ Yan Zhang $^{c}$ Liyun Zheng ${ }^{c}$ Jinhua Jiang ${ }^{c}$ Chongren Yang $^{d}$ \\ Dong Wang ${ }^{c}$ Yingjun Zhang $^{d}$ Jianglin Fan ${ }^{\mathrm{e}}$ Yifei Wang $^{\mathrm{a}, \mathrm{b}}$ \\ a Jinan University, and ${ }^{\mathrm{b}}$ National Engineering Research Center of Genetic Medicine, Guangzhou, ${ }^{\mathrm{C} H e n a n}$ Institute of \\ Medical Sciences, Zhengzhou, ${ }^{d}$ Kunming Institute of Botany, The Chinese Academy of Sciences, Kunming, China; \\ e Department of Molecular Pathology, Interdisciplinary Graduate School of Medicine and Engineering, University of \\ Yamanashi, Yamanashi, Japan
}

\section{Key Words}

Myocarditis $\cdot 20($ S)-Protopanaxtriol $\cdot$ Coxsackievirus B3

\begin{abstract}
Objective: Coxsackievirus B3 (CVB3) is a dominant causative agent for viral myocarditis. So far, effective therapies for the treatment of the disease are not available. 20(S)-Protopanaxtriol is a major component of Panax pseudoginseng and has been clinically used for the treatment of heart diseases. However, it is not known whether 20(S)-protopanaxtriol exerts any anti-viral effects. Thus, the aim of this study was to investigate the therapeutic effects of 20(S)-protopanaxtriol against CVB3 in vivo and in vitro. Methods: The antiviral effects of 20(S)-protopanaxtriol in vitro were evaluated in $\mathrm{HeLa}$ cells infected by CVB3. Then, we examined the protective effects of 20(S)-protopanaxtriol on CVB3-induced myocarditis in BALB/C mice. These mice were treated with 20(S)-protopanaxtriol at doses of $100-400 \mathrm{mg} \cdot \mathrm{kg}^{-1} \cdot \mathrm{day}^{-1}$ for 7 days and compared with the controls. Results: We found that 20(S)protopanaxtriol possessed potent antiviral effects on CVB3 in vitro. Compared with control mice, virus titers and pathological changes in the hearts were significantly decreased in the 20(S)-protopanaxtriol-treated group. Furthermore, biochemical markers of myocardial injury such as plasma lactate dehydrogenase and creatine kinase were decreased to nor-
\end{abstract}

\section{KARGER}

Fax +4161306 1234 E-Mail karger@karger.ch www.karger.com

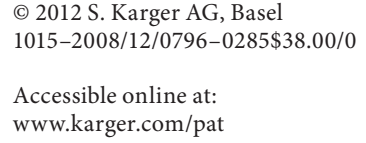

mal levels. Conclusions: These data provide the possibility that 20(S)-protopanaxtriol can be used as a potential therapeutic means for treatment of viral myocarditis.

Copyright $\odot 2012$ S. Karger AG, Basel

\section{Introduction}

Viral myocarditis has been recognized as a cause of congestive heart failure for more than 50 years, but it is still a challenging disease for diagnosis and treatment [1, 2]. The history and clinical features are often nonspecific, and practical serological markers are not available during the acute phase of the disease. Even after proper diagnosis, no clinically proven treatment exists to prevent the development of subsequent dilated cardiomyopathy and death. Many viruses have been implicated as possible causes of myocarditis. Coxsackievirus B3 (CVB3) is one of them. Recently, it has been reported that infection of the cardiac myocyte is required for the induction of cardiac dysfunction and inflammation when mice were systemically infected with CVB3 [3]. This demonstrates a crucial role of CVB3 infection of cardiac myocytes in the heart during the development of CVB3-mediated myocarditis [4].

Although much work on the development of vaccines and therapeutic agents for the treatment of myocarditis

Tel. +86 208522 3426, E-Mail twang-yf@163.com 


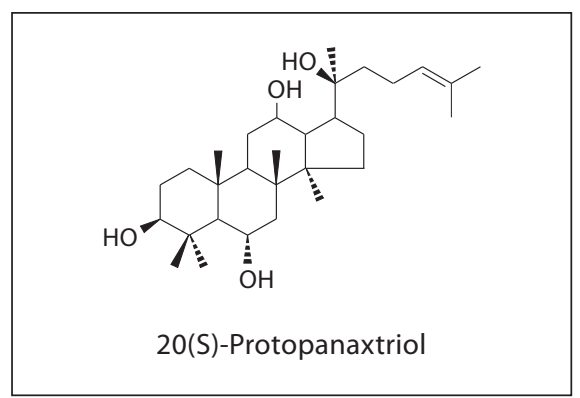

Fig. 1. Structure of 20(S)-protopanaxtriol.

has been conducted, no antiviral agents are available for clinical use. In this study, we investigated the antiviral effects of a natural extract from a traditional Chinese medicinal plant.

Panax notoginseng (Burk.) F.H. Chen is a well-known traditional Chinese medicinal plant, with the first recorded usage dating from the 16th century. It can help improve blood flow through the coronary arteries, suggesting that it can be useful for treatment of arteriosclerosis, high blood pressure and angina [5]. The roots of $P$. notoginseng are considered to contain analgesic, anti-inflammatory, antiphlogistic, antiseptic, astringent, cardiotonic, discutient, diuretic, hemostatic, hypoglycemic and styptic components [6]. 20(S)-Protopanaxtriol is one of the most important triterpene extracted from this herb. Its biological effects have been widely studied. For example, it improves cardiac function and protects myocardial cells from reperfusion injury. However, little information is available in the literature about 20(S)-protopanaxtriol effects on viral myocarditis induced by CVB3 infection. Therefore, the objective of the present study was to investigate the possible antiviral effects of 20 (S)protopanaxtriol against $\mathrm{CVB} 3$ in vitro and in vivo.

\section{Materials and Methods}

\section{Compounds}

20(S)-Protopanaxtriol ( $>97 \%$ pure) was obtained from the Laboratory of Phytochemistry, Kunming Institute of Botany, Chinese Academy of Sciences (China) [7]. The chemical structure is shown in figure 1 .

\section{Viruses and Cells}

The CVB3 Nancy strain and the HeLa cell line were generously provided by the Wuhan Institute of Virology, Chinese Academy of Sciences, Wuhan, China. HeLa cells were maintained in Dulbecco's modified Eagle medium (DMEM) supplemented with $10 \%$ fetal bovine serum, $100 \mathrm{U} / \mathrm{ml}$ penicillin, $0.1 \mathrm{mg} / \mathrm{ml}$ streptomycin, at $37^{\circ} \mathrm{C}$ in a humid atmosphere with $5 \% \mathrm{CO}_{2}$. The media used for the cytotoxic assay as well as for antiviral assays contained $2 \%$ of fetal calf serum.

\section{Animals}

Male BALB/c mice (4 weeks old, 14-16 g) were purchased and maintained at the Experimental Animal Center, Zhengzhou University (clean grade, certificate number 0009706). The animals were housed under constant conditions at a temperature of $23 \pm$ $1^{\circ} \mathrm{C}$, with $40 \pm 5 \%$ humidity, and on a 12-hour light/12-hour dark cycle. They had free access to pellet food and water.

\section{Determination of Cytotoxicity}

To determine the $50 \%$ toxic concentration $\left(\mathrm{TC}_{50}\right.$ ) of 20 (S)-protopanaxtriol, confluent HeLa cell monolayers grown in 96-well plates were incubated with serial 2 -fold dilutions of compound in $100 \mu \mathrm{l}$ test medium. The plates were incubated at $37^{\circ} \mathrm{C}$ and checked daily by phase contrast microscopy. After $48 \mathrm{~h}$ of incubation, $20 \mu \mathrm{l}$ MTT [(3-(4,5-dimethylthiazol-2-yl)-2,5-diphenyl tetrazolium bromide)] $(0.5 \mathrm{mg} / \mathrm{ml})$ was added to each well and allowed to react for $4 \mathrm{~h}$. After removal of the supernatant, $100 \mu \mathrm{l}$ of DMSO was added to each well. Plates were incubated at room temperature for $30 \mathrm{~min}$ and the optical density was measured at the dual wavelengths of 570 and $630 \mathrm{~nm}$. $\mathrm{TC}_{50}$ was defined as the concentration of compound required to reduce the viability of untreated cell cultures by $50 \%$. The mean dose-response curve of at least three tests was used to calculate the $\mathrm{TC}_{50}$.

\section{Antiviral Activity}

HeLa cell monolayers were grown in 96-well flat-bottomed microtiter plates. After removal of culture medium, $50 \mu \mathrm{l}$ of drug solution and a constant amount of $10050 \%$ tissue culture infective dose $\left(\mathrm{TCID}_{50}\right)$ CVB3 in a volume of $50 \mu \mathrm{l}$ was added to the cells. After $24 \mathrm{~h}, 20 \mu \mathrm{l}$ MTT $(0.5 \mathrm{mg} / \mathrm{ml})$ was added to each well and allowed to react for $4 \mathrm{~h}$. After removal of the supernatant, $100 \mu \mathrm{l}$ of DMSO was added to each well. Plates were incubated at room temperature for $30 \mathrm{~min}$ and the optical density was measured at the dual wavelengths of 570 and $630 \mathrm{~nm} . \mathrm{IC}_{50}$ is the concentration of the drug required to inhibit $50 \%$ of the viral cytopathic effect. The mean dose-response curve of at least three tests was used to calculate the $\mathrm{IC}_{50}$.

\section{In vivo Antiviral Activity Experiment - Experiment Design and Antiviral Therapy}

In this study, 90 mice were inoculated intraperitoneally with CVB3 (100 TCID 50$)$. Forty-five of the inoculated mice received $20(\mathrm{~S})$-protopanaxtriol orally at doses of $100(n=15), 200(n=15)$ and $400 \mathrm{mg} \cdot \mathrm{kg}^{-1} \cdot \mathrm{day}^{-1}(\mathrm{n}=15)$ for 7 days. The doses were set based on our observation that three doses of 20(S)-protopanaxitriol (100, 200 and $400 \mathrm{mg} / \mathrm{kg} / \mathrm{day}$ ) had no toxicity in the mouse (data not shown). Oral administration with $150 \mathrm{mg} \cdot \mathrm{kg}^{-1} \cdot \mathrm{day}^{-1}(\mathrm{n}=15)$ of ribavirin (RBV) was used as a positive control. CVB3-inoculated mice $(n=15)$ were treated with a $0.9 \% \mathrm{NaCl}$ solution daily and used as infected controls. Uninoculated mice $(n=15)$ were treated with an oral dose of $0.9 \%$ saline solution daily and used as normal control. Mice were sacrificed on the 7th day. Blood was collected retro-orbitally and the serum was separated to measure the activities of lactase dehydrogenase ( $\mathrm{LDH})$ and creatine kinase $(\mathrm{CK})$ by using commercially available kits (Nanjing Jiancheng Bioengineering Institute, Nanjing, China) to analyze myocardial injury. The heart 


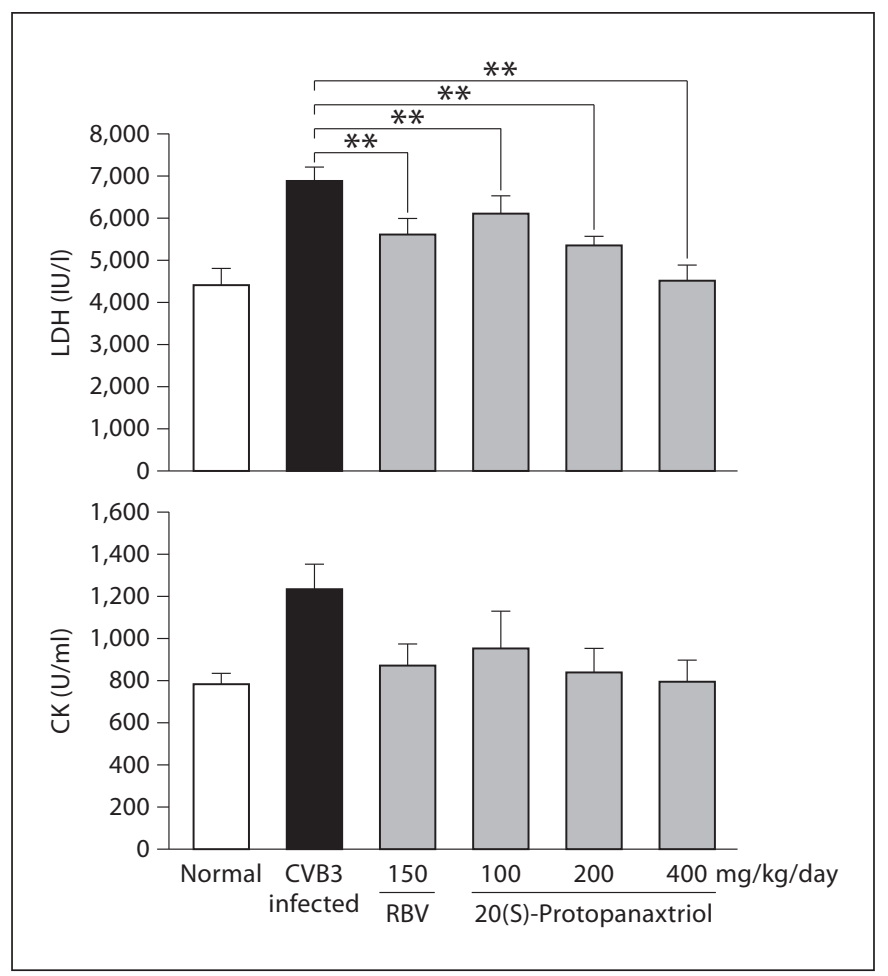

Fig. 2. 20(S)-Protopanaxtriol decreases LDH and CK levels in CVB3-infected mice. Mice were treated with RBV (as positive control) and different doses of 20(S)-protopanaxtriol after CVB3 infection. Serum levels of $\mathrm{LDH}$ and CK were measured at day 7. $n=15$ for each group. ${ }^{* *} \mathrm{p}<0.01$ versus the CVB3-infected group.

Table 1. Cytotoxic effects of 20(S)-protopanaxtriol on HeLa cells and antiviral activity against CVB3

\begin{tabular}{lrcr}
\hline Drug & $\mathrm{TC}_{50}, \mu \mathrm{g} / \mathrm{ml}$ & $\mathrm{IC}_{50}, \mu \mathrm{g} / \mathrm{ml}$ & \multicolumn{1}{l}{$\mathrm{TI}$} \\
\hline 20(S)-Protopanaxtriol & $28.77 \pm 3.61$ & $2.74 \pm 0.33$ & $10.56 \pm 1.59$ \\
Ribavirin & $722 \pm 66.2$ & $190 \pm 21.66$ & $3.81 \pm 0.09$ \\
\hline
\end{tabular}

$\mathrm{TI}=$ Therapeutic index $=\mathrm{TC}_{50} / \mathrm{IC}_{50}$.

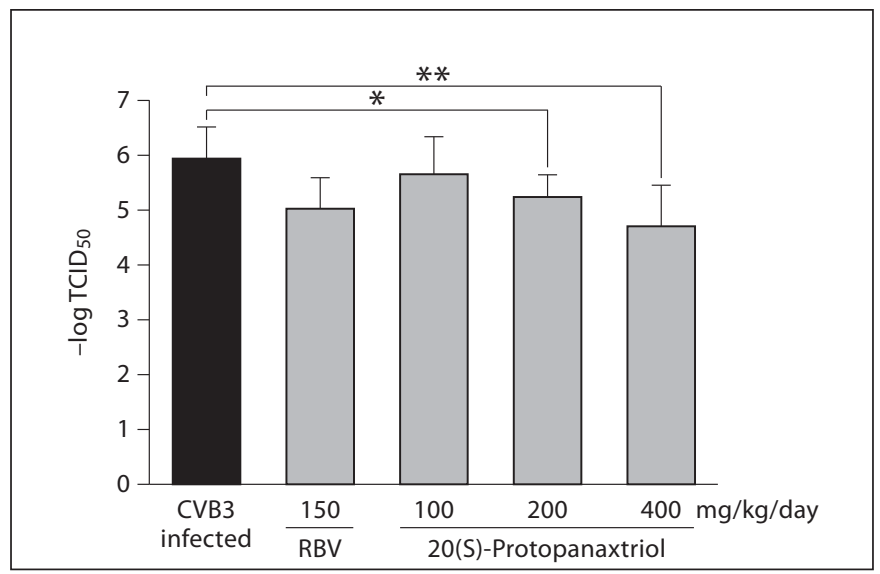

Fig. 3. Virus titers of the hearts of each group. Mice were sacrificed at day 7 , and the hearts were homogenized and virus titers were determined as described in Materials and Methods. $\mathrm{n}=15$ for each group. ${ }^{*} \mathrm{p}<0.05,{ }^{* *} \mathrm{p}<0.01$ versus the CVB3-infected group.

\section{Histology}

Histology was performed to examine the state of myocardial injury. The hearts were dissected from the mice, fixed in $10 \%$ formalin solution, embedded in paraffin, sectioned serially to a thickness of $3 \mu \mathrm{m}$, and stained with hematoxylin-eosin (HE). HEstained sections were blindly and independently examined under a light microscope for evaluation of myocarditis.

\section{Electron Microscopy}

Myocardial injury was further examined by transmission electron microscopy. Heart samples were fixed in $4 \%$ formaldehyde and $1 \%$ glutaraldehyde. After dehydration, embedding, sectioning and staining, these sections were observed under a JEOL 200CX transmission electron microscope (Tokyo).

\section{Statistical Analyses}

Data are expressed as the mean \pm SD. One-way analysis of variance with unpaired Student's t test was used to determine group differences. For all data, $\mathrm{p}<0.05$ was considered statistically significant.

\section{Results}

was divided into two parts: one part was homogenized in DMEM to determine virus titers by TCID $_{50}$ assays, and the other part was fixed for histological and electron microscopy analysis.

Virus Titers of the Heart

Mice were sacrificed 8 days after infection. The hearts were removed and washed with physiological saline, then homogenized in DMEM containing $4 \%$ fetal calf serum. The cellular debris was removed by centrifugation at $2,000 \mathrm{~g}$ for $10 \mathrm{~min}$ at $4{ }^{\circ} \mathrm{C}$. Virus titers were measured in 10 -fold serial dilutions of the supernatant in DMEM by TCID 50 assays in HeLa cells.

Protective Effects of 20S-Protopanaxtriol on CVB3-Induced Myocarditis

\section{0(S)-Protopanaxtriol Has Potent Anti-CVB3 Activity in vitro}

The cytotoxicity and antiviral activity of 20(S)-protopanaxtriol against CVB3 were examined in HeLa cells. As shown in table $1, \mathrm{TC}_{50}$ of $\mathrm{RBV}$ and $20(\mathrm{~S})$-protopanaxtriol on HeLa cells were 722 and $29 \mu \mathrm{g} / \mathrm{ml}$, respectively. Both 20(S)-protopanaxtriol and RBV inhibited the replication of CVB3 in HeLa cells. The $\mathrm{IC}_{50}$ for inhibition of CVB3 replication in HeLa cells was $190 \mu \mathrm{g} / \mathrm{ml}$ for RBV 

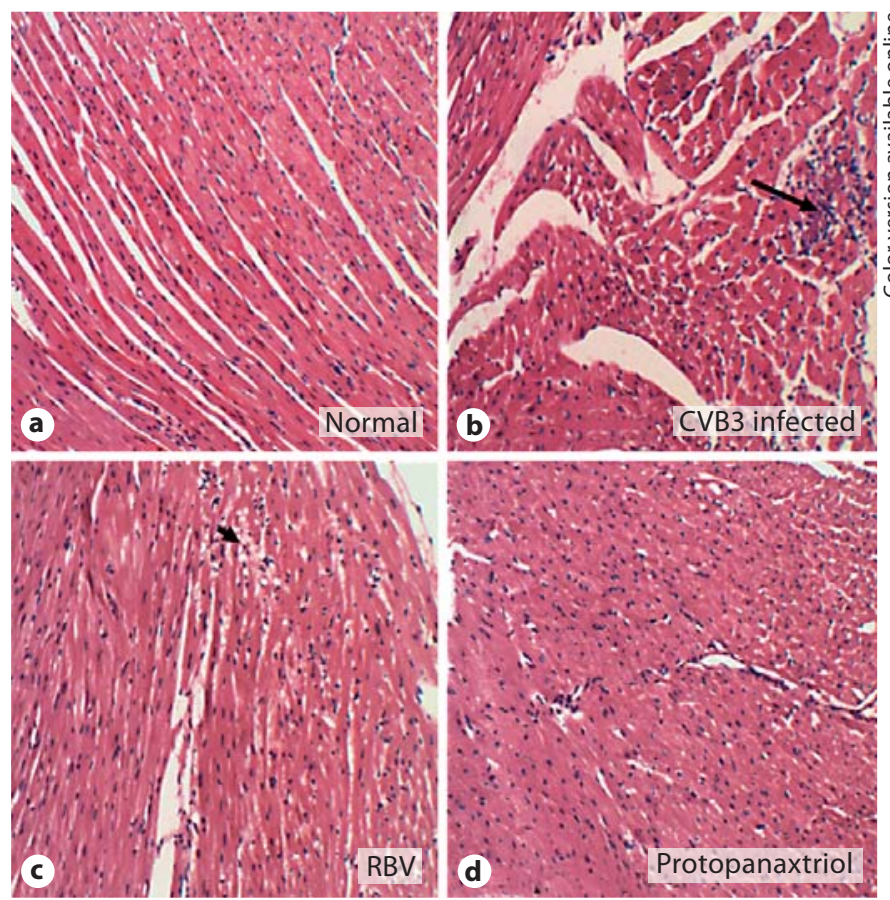

Fig. 4. Histological features of CVB3-induced myocarditis. Representative micrographs (HE staining) from each group are shown. The arrow indicates the foci of mononuclear cell infiltration, and the arrowhead indicates myocardial necrosis. $\times 400$.

and $2.74 \mu \mathrm{g} / \mathrm{ml}$ for $20(\mathrm{~S})$-protopanaxtriol, respectively, indicating that the inhibitory effects of 20(S)-protopanaxtriol against CVB3 were stronger than that of RBV.

\section{0(S)-Protopanaxtriol Decreases LDH and CK Levels} in CVB3-Infected Mice

Infection of CVB3 resulted in myocarditis in mice as indicated by increased plasma levels of $\mathrm{LDH}$ and CK (fig. 2). However, treatment with 20(S)-protopanaxtriol led to an obvious decrease in the activities of LDH and $\mathrm{CK}$ in infected mouse serum compared with control groups.

\section{0(S)-Protopanaxtriol Inhibits CVB3 Replication and}

CVB3-Induced Myocarditis in Mice

Virus titers of the hearts in the infected mice were clearly decreased after treatment with 20(S)-protopanaxtriol at doses of 200 and $400 \mathrm{mg} \cdot \mathrm{kg}^{-1} \cdot \mathrm{day}^{-1}$ (fig. 3). Histological examination revealed that CVB3 infection induced focal myocardial necrosis accompanied by mononuclear cell infiltration, which is compatible with the histological features of myocarditis (fig. 4). Treatment with either RBV or 20(S)-protopanaxtriol or apparently
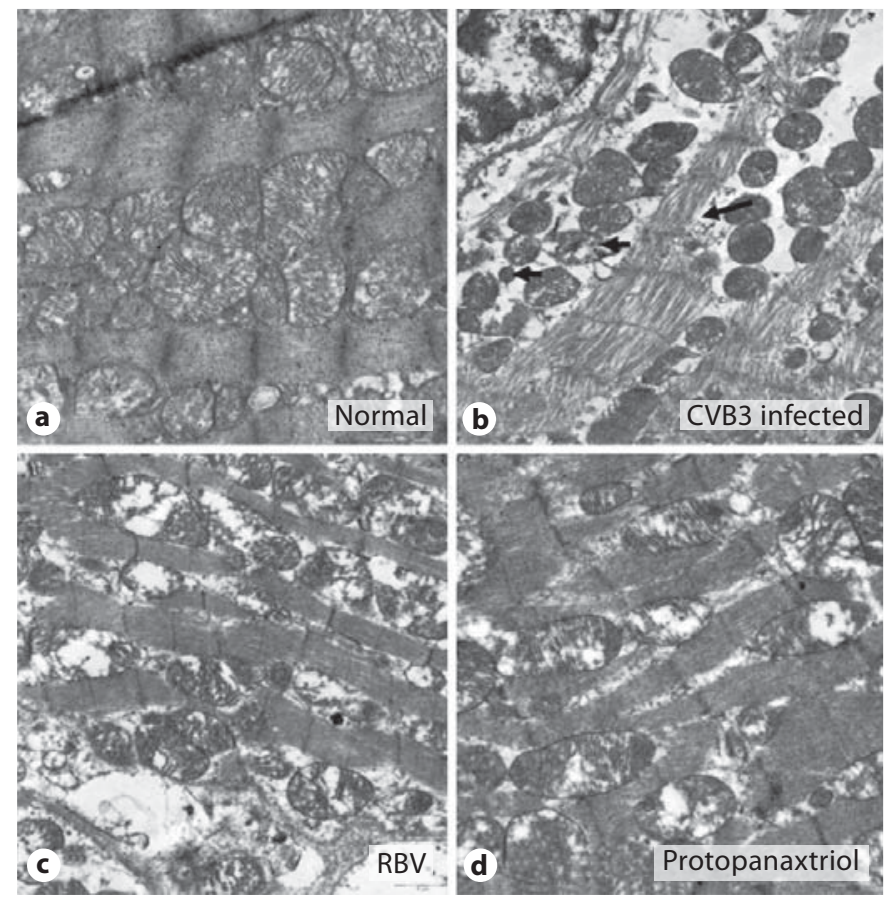

Fig. 5. Ultrastructural features of CVB3-induced myocarditis. In the CVB3-infected group, the cardiac muscles were in disarray, and sarcomere fragmentation was observed (arrow). In addition, mitochondria had shrunk (arrowheads). $\times 10,000$.

reduced mononuclear cell infiltration and the myocardium damage was relieved.

Ultrastructural changes in the myocardium were further examined by transmission electron microscopy. In the CVB3-infected group, the cardiac muscle was in disarray, and sarcomere fragmentation was observed. In addition, mitochondria were shrunken (fig. 5). Treatment with the 20(S)-protopanaxtriol group led to the reduction of these changes, similarly to the treatment of RBV.

\section{Discussion}

Although the exact molecular mechanism responsible for viral myocarditis is not completely clarified, the cardiac damage caused by CVB3 is considered to be mediated by the following mechanisms, including autoimmune reaction [8], direct cytotoxicities [9] and virusinduced apoptosis [10-12]. In the clinical sites, immunosuppressive agents (azathioprine, prednisone and cyclosporine), intravenous immunoglobulin and antiviral agents (interferon, pleconaril and acyclovir) are often 
used for treatment of the disease [13]. However, most of these treatments are for improving symptoms. Recently, effective ingredients isolated from traditional Chinese herbs have been shown to have antiviral activities including CVB3 [14, 15]. 20(S)-Protopanaxtriol is the major component isolated from the roots of $P$. notoginseng, yet its biological effects on myocarditis have not been fully studied. In the present study, we found that 20(S)-protopanaxtriol has potent anti-CVB3 activities both in vitro and in vivo and can significantly improve CVB3-induced myocarditis in mice.

Of note, the therapeutic index of 20(S)-protopanaxtriol on CVB3-infected HeLa cells was higher than that of RBV. In addition, our study showed that 20(S)-protopanaxtriol, when administered to a murine model of viral myocarditis, not only markedly decreased the virus titers in serum, but also protected against CVB3-induced cardiac injury and inflammatory cell infiltration. Throughout the in vitro experiment, the cytotoxicity of 20(S)-protopanaxitriol seemed to be higher than that of RBV, and even high doses by oral administration are not only effective but also safe in vivo [16]. The finding of reduced cardiac necrosis and mononuclear cell infiltration after treatment with 20(S)-protopanaxtriol suggests that 20(S)protopanaxtriol may have several therapeutic functions against CVB3-induced myocarditis in vivo. Several mechanisms may be operative for anti-viral effects of 20(S)-protopanaxtriol. For example, 20(S)-protopanax- triol may be directly involved in an anti-inflammatory process caused by CVB3 infection, or may protect myocardiocytes from cell death (apoptosis). In our preliminary study, treatment with 20(S)-protopanaxtriol for $24 \mathrm{~h}$ significantly prevents from HeLa cell apoptosis induced by CVB3 infection (data not shown). This notion is further supported by the observation that biochemical markers including CK and LDH used to identify myocardial injury were normalized in 20(S)-protopanaxtrioltreated mice. It is possible that $20(\mathrm{~S})$-protopanaxtriol protected myocardial cells from the harmful effects of CVB3 by decreasing the leakage of the enzymes from the myocardial cells into the blood stream. In spite of this, the molecular mechanisms for 20(S)-protopanaxtriol anti-viral effects remain to be disclosed in future.

In conclusion, our study showed that 20(S)-protopanaxtriol had antiviral effects on CVB3 virus-induced myocarditis in mice. These findings suggest that 20 (S)protopanaxtriol may be potentially useful for the treatment of patients suffering from viral myocarditis.

\section{Acknowledgements}

This study was supported by the Joint Funds of the National Science Foundation of China (U0632010), the State Key Laboratory of Phytochemistry and Plant Resources in West China, Chinese Academy of Sciences (O807B11211, O807E21211), and '211 grant of MOE'.

\section{References}

1 Gupta S, Markham DW, Drazner MH, Mammen PP: Fulminant myocarditis. Nat Clin Pract Cardiovasc Med 2008;5:693-706.

$\checkmark 2$ Peters NS, Poole-Wilson PA: Myocarditis: continuing clinical and pathologic confusion. Am Heart J 1991;121:942-947.

- 3 Shi Y, Chen C, Lisewski U, Wrackmeyer U, Radke M, Westermann D, Sauter M, Tschöpe C, Poller WC, Klingel C, Gotthardt M: Cardiac deletion of the Coxsackievirus-adenovirus receptor abolishes Coxsackievirus B3 infection and prevents myocarditis in vivo. J Am Coll Cardiol 2009;53:1219 -1226.

4 Yajima T, Knowlton KU: Viral myocarditis: from the perspective of the virus. Circulation 2009;119:2615-2624.

5 Chevallier A: The Encyclopedia of Medicinal Plants. London, Dorling Kindersley, 1996.

6 Duke JA, Ayensu ES: Medicinal Plants of China. Algonac, Reference Publications, 1985.

7 Liao PY, Wang D, Zhang YJ, Yang CR: Dammarane-type glycosides from the steamed notoginseng. J Agric Food Chem 2008;56: 1751-1756

8 Cihakova D, Rose NR: Pathogenesis of myocarditis and dilated cardiomyopathy. Adv Immunol 2008;99:95-114.

-9 Kearney MT, Cotton JM, Richardson PJ, Shah AM: Viral myocarditis and dilated cardiomyopathy: mechanisms, manifestations, and management. Postgrad Med J 2001;77: 4-10.

10 Yuan JP, Zhao W, Wang HT, Wu KY, Li T, Guo XK, Tong SQ: Coxsackievirus B3-induced apoptosis and caspase-3. Cell Res 2003;13:203-209.

-11 Martin U, Jarasch N, Nestler M, Rassmann A, Munder T, Seitz S, Zell R, Wutzler P, Henke A: Antiviral effects of pan-caspase inhibitors on the replication of coxsackievirus B3. Apoptosis 2007;12:525-533.

12 Carthy CM, Yanagawa B, Luo H, Granville DJ, Yang D, Cheung P, Cheung C, Esfandiarei M, Rudin CM, Thompson CB, Hunt DW,
McManus BM: Bcl-2 and Bcl-xL overexpression inhibits cytochrome $\mathrm{c}$ release, activation of multiple caspases, and virus release following coxsackievirus B3 infection. Virology 2003;313:147-157.

13 Brunetti L, DeSantis ER: Treatment of viral myocarditis caused by coxsackievirus B. Am J Health Syst Pharm 2008;65:132-137.

14 Zhu H, Zhang Y, Ye G, Li Z, Zhou P, Huang $\mathrm{C}$ : In vivo and in vitro antiviral activities of calycosin-7-O-beta-D-glucopyranoside against coxsackievirus B3. Biol Pharm Bull 2009;32:68-73.

15 Wang H, Ding Y, Zhou J, Sun X, Wang S: The in vitro and in vivo antiviral effects of salidroside from Rhodiola rosea $\mathrm{L}$. against coxsackievirus B3. Phytomedicine 2009;16:146-155.

16 Wang YZ, Wang YS, Chu SF, Chen NH, Zhang JT: Protopanaxatriol metabolites identified by LC-MS/MS after oral administration in mice. Int J Clin Pharmacol Ther 2010;48:282-290. 